Sibylle Benninghoff-Lühl • »Figuren des Zitats« 


\section{Sibylle Benninghoff-Lühl}

\section{»Figuren des Zitats «}

Eine Untersuchung zur Funktionsweise übertragener Rede

Verlag J.B. Metzler

Stuttgart · Weimar 
Die Deutsche Bibliothek - CIP-Einheitsaufnahme

\section{Benninghoff-Lühl, Sibylle:}

Figuren des Zitats : eine Untersuchung zur Funktionsweise übertragener Rede / Sibylle Benninghoff-Lühl. - Stuttgart ; Weimar : Metzler, 1998

ISBN 978-3-476-45201-6

ISBN 978-3-476-45201-6

ISBN 978-3-476-04295-8 (eBook)

DOI 10.1007/978-3-476-04295-8

Dieses Werk ist einschließlich aller seiner Teile geschützt. Jede Verwertung außerhalb der engen Grenzen des Urheberrechtsgesetzes ist ohne Zustimmung des Verlages unzulässig und strafbar. Das gilt insbesondere für die Vervielfältigungen, Übersetzung, Mikroverfilmungen und Einspeicherung in elektronischen Systemen.

M \& P Schriftenreihe für Wissenschaft und Forschung

(C) 1998 Springer-Verlag GmbH Deutschland

Ursprünglich erschienen bei J.B. Metzlersche Verlagsbuchhandlung und Carl Ernst Poeschel Verlag GmbH in Stuttgart 1998 
Die vorliegende Untersuchung wurde als Habilitationsschrift im Fach Neuere Deutsche Literatur von der Philosophischen Fakultät II der Humboldt-Universität zu Berlin im November 1997 angenommen. Für die Drucklegung der Arbeit habe ich den Stand der Forschung über Hinweise in den Fußnoten soweit wie möglich aktualisiert.

Mein Dank gilt vor allem den Betreuern der Habilitationsschrift, Frau Prof. Dr. Inge Stephan und Herr Prof. Dr. Hartmut Böhme.

Sie haben mir von Anfang nicht nur mit ihren Gutachten, sondern auch mit vielen Anregungen, mit Kritik und beharrlicher Ermutigung geholfen.

Mein Dank geht weiterhin an Mascha Braun, Claudia Böttger, Walter Hellmann, Liselotte Hermes da Fonseca, Ines Rüge, Barbara Sievers und Christiane Tomfohrde. Sie haben mir mit vielen Hinweisen, Informationen und Ratschlägen zur Seite gestanden.

Gefördert wurde die Untersuchung durch ein Habilitandenstipendium der Deutschen Forschungsgemeinschaft. 
Meiner Familie gewidmet 
Zitieren und Wissenschaftssprache. Verschiedene Herangehensweisen an das Thema: Geschichtsschreibung, Intertextualität, Interdisziplinarität. Sammeln von Gesichtspunkten, die in Zitatdefinitionen auftauchen: Wie funktionieren Zitatdefinitionen? Übertragungen, Übersetzungen des Begriffs, Metaphern, Figuren im engeren und im übertragenen Sinn. Was sind Metaphern, was Figuren? Antike Rhetorik, die zwischen nackter, gemeiner, reiner Sprache und zwischen eingekleideter und ausgeschmückter Rede unterscheidet. Zitat: Schmuck und Gewürz. Relektüre der rhetorischen Figuren: Die Unentscheidbarkeit von eigentlicher (natürlicher), väterlicher und übertragener Rede. Problematik einer ,Meta' ${ }^{6}$-Sprache. Kann man der Metapher entkommen? Bemerkungen zur ,Metaphorologie‘. Materialauswahl und Anordnung der Untersuchung.

Literatur- und kulturwissenschaftliche Texte zum Zitat, zur Metapher, zur Figur; Aristoteles, Quintilian, Cicero; Antoine Compagnon, Claudette Sartiliot, Dubravka Oraić Tolić; Roland Barthes, Jacques Derrida und Paul de Man zur Metapher und Figur

\section{B. Der Freund}

Das Zitat als Effekt einer sehr engen, innigen Beziehung zwischen den Texten. Kommentare des Urheberrechts zum Zitieren als „Fair-Play“. Der Freund - eine aufrechte Figur, die ins Kippen gerät. Autoritätszitat. Zitierkartelle. Mit Zitaten kämpfen. In den Bann geschlagen sein von (Eingangs-)Zitaten. Außer Gefecht setzen: Feind? Richtiges und falsches Zitieren, vorschreiben, angeben; Anruf des toten Freundes. Apostrophe: Anpfiff, barsches Anfahren, eine Schuld haben, Rechnung ausstellen, begleichen bzw. offen haben, in der Schuld stehen, handeln. 
Texte zur Geschichte und Theorie des ,Zitats'. Paul de Man: Autobiographie als Maskenspiel, Epistemologie der Metapher, Allegorien des Lesens. Jacques Derrida: Mémoires I und II. Politiques de l'amitié, Feuer und Asche

\section{Der Verräter}

Zitieren, in Bewegung versetzen, erregen, wecken über ein Herbeirufen. Anführen. Einen Vortext verraten, spionieren, von einem in den anderen Text sehen.

Jacques Derrida, Roland Barthes, Claudette Sartiliot, Antoine Compagnon, Paul de Man; lexikalisch-etymologische Texte

\section{Ein Original}

Assimilation/Dissimilation. Entlehnen, verweisen, anmerken, auszeichnen, mit Fußnoten versehen. Die gelehrte Fußnote.

Herman Meyer „Das Zitat in der Erzählkunst“

\section{E. Geflügeltes Wesen}

Worte/Namen in Umlauf bringen, aus der Luft greifen, Flügel verleihen, einen Ursprung verbuchen und verzeichnen. Apostrophierung der ,Quelle‘ Georg Büchmann, Franz Kafka, Lexika der Geflügelten Worte

\section{F. Räuber, Schwindler, Menschenfresser}

Wiederholen, verkleiden, verschlingen, aus dem Zusammenhang reißen, erinnern, vergessen, aufschieben der Katastrophe. Siegel und Name. Einmaligkeit und Plagiat.

Walter Benjamin, Bettine Menke, Jacques Derrida 
Listen, Serienbildung, Montage, Collage: fügen, reihen, knüpfen, kleben, haften, aufziehen, ordnen, klassifizieren, beschreiben, schneiden. In ein Programm zwingen, aus der Ordnung herausspringen: „Das Programm einer Reihe macht Jagd“". Gesichter über eine zugefallene Nachbarschaft annehmen. Zitat als Gelenk/Scharnier; Zitate zähmen, bändigen.

Literaturwissenschaftliche Texte zum Zitat als Fremdkörper, Writing Culture, Protokolle der Verhandlungen der Gesellschaft für Ethnologie, Dan Sperber, Michel Foucault, Jacques Derrida, Paul de Man, Roland Barthes

\section{H. Schatzsucher, Blütenleser, Steinbrecher, Quellenforscher}

Aus dem Steinbruch hauen, Edelstein, Perle, facettieren, anverwandeln, schleifen, bilden, glänzen, krönen, verlieren, suchen, graben, finden, lustvolles Wiedererkennen. Wörterbücher, Wortschatz, Schatzkästchen, Thesaurus, Wert, Münzen, Schatzgräber, Wünschelrutengänger, Auflesen, Lesefrüchte und Stilblüten sammeln, eine Lese betreiben, einer Stelle/Quelle hinterhersein, aufstören, aufstöbern, stoßen auf, zielsicher treffen, aufspieBen, pressen, konservieren, ausstellen, verlesen, abbrechen, wuchern, ranken, aufpfropfen, veredeln, streuen, fruchtbar machen; erlesen, naturwüchsig; Gestrüpp, Unkraut. Gärten anlegen.

Jean Paul, Eduard Berend, Herman Meyer, Walter Rehm, Götz Müller, Gustav Lohmann, Jacques Derrida: Die weiße Mythologie 


\section{Urheber, Schöpfer, Geist}

Sehen und erscheinen, anrufen, sich berauschen, halluzinieren, das Abwesende anreden, besprechen, beschwören, hervorzaubern, abwenden, blind sehen, blinde Stellen lesen, Unlesbarkeit. Wie fällt ein Zitat ein? An welcher Stelle und warum? Etwas wirkt ,weit herbeigeholt', ,An den Haaren herbeigezogen'. Wie sind die Verbindungen zum bereits einmal Gelesenen, Gewesenen? Wie ist das Verhältnis von alt und neu? Wie funktioniert ein Geist? Urhebergesetz: Schöpfer als geistiges Wesen, der ,Neues' individuell und selbständig gestaltet: Kein Tier, keine Maschine; Psychoanalyse: Zitieren als Umgehen bzw. Brechen von Widerstand. Identifizieren, Wiederholen, Verschieben des Ursprungs.

Urhebergesetz, Kommentare zum Urhebergesetz, Johann Wolfgang Goethe, Sigmund Freud, Goethe Zitate in der Goethe-Preisrede von S. Freud 1930, Walter Schönau

\section{J. Spieler}

Anagrammatisches wiederholen, vertauschen, wetten, auf eine Stelle setzen, würfeln, hingerissen sein, aussetzen, zufällig gewinnen, Kopf-undKragen verlieren, eine Chance haben/vergeben, eine Wahl treffen. Spielarten des Zitierens. Zitieren als ,Playing the game'. Anspielungen, Allusion. Beispiele. Mit Zitaten spielen. Zitierhäufigkeit und Spielmarken.

Jacques Derrida, Walter Benjamin, Texte zur "Unsinnspoesie“ und Anagrammatik. Texte zur Theorie der Zitationsanalyse. J.W. Goethe: „Verweile nur ... “, Stellen zur Wette in Faust I/II 


\section{K. Enthüllung des Falls}

Zur Unentscheidbarkeit zwischen eigentlicher und übertragener Rede im Fall der Kategorie ,Zitat'. Bedeutung des ,Nackten' für die ,Enthüllung'. Ursprung, Quelle als Effekt der Apostrophierung. Anregung, das Ein- und Aussetzen der Bewegung, sowie ihren Verlauf im Reflektieren der Figuren des Zitats mitzuartikulieren. Zusammenfassung und Ausblick auf Figuren, welche die Rede über das Zitat ins Spiel bringt. Noch einmal: Rhetorische Figuren sowie Zusammenhang von Figuration/Defiguration, Ähnlichkeit/Unähnlichkeit.

\section{Bibliographie}

